\title{
Psychopathologie in der forensischen Psychiatrie
}

\author{
Hans-Ludwig Kröber
}

Online publiziert: 24. September 2010

(C) Springer-Verlag 2010

Forensische Psychiatrie ist ein eigentümliches Teilgebiet der Psychiatrie: Es ist ein Spezialgebiet, das aber voraussetzt, dass die hier Tätigen Generalisten des Faches sind, dass sie also besonders umfangreiche Erfahrungen in der Diagnostik und Krankheitskunde sämtlicher psychischer Störungen haben, weit hinein in die Übergangsbereiche zum Normalen. Hinzu kommen eingehende kriminologische Kenntnisse und strafrechtliches Wissen. Kernbereich der Psychiatrie ist die Psychopathologie, die Lehre von den Erscheinungsformen seelischer Störung und Krankheit. Insofern ist auch die Unterscheidung zwischen normalpsychologischen Abläufen einerseits (wie beispielsweise einer heftigen Affekterregung nach entsprechender Belastung) und psychopathologischen Phänomenen andererseits (z. B. toxisch verursachter Verlangsamung der Denkabläufe) ein wesentlicher Teil der professionellen Befassung in der Diagnostik und Behandlung von Rechtsbrechern und Zeugen. Das Handbuch der Forensischen Psychiatrie wird im noch fehlenden 2. Band, der inzwischen beim Verlag ist, eingangs ein großes Kapitel zur Psychopathologie enthalten.

Allerdings ist durchaus auch zu prüfen, wie viel Psychopathologie überhaupt noch im Fach steckt, und ob nicht so etwas wie eine klinische Kriminologie inzwischen dominiert, mit entsprechend eher sozialdiagnostischen Vorgaben und Entscheidungen, wie sie nicht zuletzt bei der Kriminalprognostik erhebliches Gewicht haben (z. B. Art und Formen der bisherigen Delinquenz, Formen der bisherigen sozialen Integration, Möglichkeiten sozial stützender Maßnahmen im Empfangsraum etc.). Die Relevanz der Psychopathologie war Thema der 14. Berliner Junitagung für

Prof. Dr. med. H.-L. Kröber $(\bowtie)$

Institut für Forensische Psychiatrie,

Charité - Universitätsmedizin Berlin,

Oranienburger Str. 285, 13437 Berlin, Deutschland

E-Mail: Hans-Ludwig.Kroeber@charite.de
Forensische Psychiatrie und Psychologie am 11.06.2010, und dieses Heft enthält fünf der dort gehaltenen Beiträge. Ein weiterer Beitrag von Hans Schanda zu den Beziehungen von Gewalttätigkeit zur Psychopathologie der Psychosen wird in einem späteren Heft folgen. Bereits die Vorträge der Junitagung waren dem wie stets anwesenden Professor Werner Janzarik gewidmet, der am 03.06.2010 sein 90. Lebensjahr vollendet hatte. Er hat als langjähriger Direktor der Heidelberger Psychiatrischen Universitätsklinik die forensisch-psychiatrische Tradition der Klinik bewahrt und intensiviert; viele der heute aktiven forensischen Psychiater sind seine Schüler oder von ihm beeinflusst. Denn Janzarik hat mit großer Ausdauer immer wieder die Zusammenhänge zwischen Erfahrungen, Einstellungen und Wertgefüge, deren normalpsychologischer Beeinflussung und psychopathologischer Gestörtheit erkundet und diese Phänomene zu einer psychiatrischen Fundierung von Rechtsbegriffen wie Einsicht, Einsichtsfähigkeit, Wollen, Hemmung, Steuerung und Steuerungsfähigkeit genutzt. Die Themen der Tagung und auch dieses Heftes stehen mit dem Werk Janzariks in Zusammenhang: Henning Saß behandelt das Problem der dauerhaften Verformung seelischer Struktur am Beispiel des Querulantenwahns und unterscheidet verstehbare von pathologischen Entwicklungen nicht zuletzt unter Rückgriff auf die Strukturdynamik. Der Unterzeichner hat sich mit der Frage befasst, warum in Deutschland bipolar Manische so selten mit Verbrechen in Erscheinung treten, wenn man doch gerade „Enthemmung“ für typisch kriminogen hält. Die Überprüfung führt zu der Annahme, dass Persönlichkeit und individuelles Wertgefüge, nicht zuletzt aber auch die Fähigkeit zur sozialen Bezugnahme, ungleich relevanter sind als eine nachlassende Steuerungsfähigkeit. Paul Hoff aktualisiert den Diskurs um die „Affektdelikte“ und fokussiert auf die Intentionsbildung und die Abwägungsprozesse in der Vorgeschichte der Tat. Matthias Lammel warnt mit 
triftigen Argumenten vor der Pathologisierung sozialer Devianz und normalpsychologischer Abläufe bei kriminellen Jugendlichen, wobei er verdeutlicht, dass auch hier strukturdynamische Überlegungen Klarheit schaffen. Elmar Habermeyer et al. widmen sich dann schließlich der Frage, wie viel Psychopathologie bei kriminalprognostischen Fragestellungen erforderlich ist.
Ergänzend zum Schwerpunktthema enthält das Heft einen Bericht über die nur geringen Effekte von Antigewalttraining bei jugendlichen Straftätern und einen Beitrag über die deutlich stärkeren Wirkungen der Entziehungsbehandlung gemäß § 64 StGB auf die Rückfälligkeit.

H.-L. Kröber 